\title{
EFFICACY OF PROXIMAL FEMORAL NAIL IN PROXIMAL FEMORAL FRACTURES
}

\author{
Prakash Wali ${ }^{1}$ Santosh Mared $^{2}$
}

${ }^{1}$ Associate Professor, Department of Orthopaedics, Belagavi Institute of Medical Sciences, Belagavi.

${ }^{2}$ Senior Resident, Department of Orthopaedics, Belagavi Institute of Medical Sciences, Belagavi.

\section{ABSTRACT}

\section{BACKGROUND}

Fractures of the subtrochanteric region of femur can be difficult to treat. This anatomic region experiences the highest tensile and compressive stresses in the human skeleton. The proximal fragment is often quite short, offering limited opportunity for internal fixation, which must withstand these substantial forces. In subtrochanteric fracture the proximal fragment is flexed, abducted and externally rotated due to the iliopsoas, abductor muscles and short external rotators muscle pull. Hence, it is useful to perform an open reduction of the fracture fragments and then stabilise the proximal fragment to restore anatomical position prior to nail insertion.

\section{MATERIALS AND METHODS}

The observational study consists of 20 adult patients with proximal femoral fractures of femur who were treated with PFN at BIMS, Belgaum, between Jan 2014 and March 2017. The fractures were classified according to Russell and Taylor classification. Twenty cases were followed at regular intervals. This study was conducted with due emphasis for clinical observation and analysis of results after surgical management of proximal femoral fractures of femur with proximal femoral nail.

\section{RESULTS}

In our study of subtrochanteric fractures treated with PFN, we encountered intraoperative complications like iatrogenic fracture of lateral cortex in 2 cases (10\%) due to selection of wrong entry point, failure to put derotation screw in 2 cases (10\%) and guidewire breakage in 1 case (5\%). Delayed complications like hip joint stiffness in 3 cases (15\%), knee joint stiffness in 1 case (5\%) and shortening of more than $1 \mathrm{~cm}$ in 1 case (5\%) and delayed union in 2 cases $(10 \%)$.

\section{CONCLUSION}

Open reduction offer advantage in preventing malalignment of subtrochanteric fractures as compared to closed reduction. From our study, we conclude that PFN is a reliable implant for subtrochanteric fractures leading to high rate of bone union restoring the anatomical alignment and reduced chance of implant failure or deformities.

\section{KEYWORDS}

Advantages of open reduction in subtrochanteric fractures.

HOW TO CITE THIS ARTICLE: Wali P, Mared S. Efficacy of proximal femoral nail in proximal femoral fractures. J. Evolution Med. Dent. Sci. 2017;6(81):5702-5706, DOI: 10.14260/jemds/2017/1237

\section{BACKGROUND}

Proximal femoral fractures occur typically at the junction between trabecular bone and cortical bone where the mechanical stress across the junction is highest in the femur, which is responsible for their frequent comminution. ${ }^{1}$ These fractures account for $10 \%$ to $34 \%$ of all hip fractures.

These fractures occur typically in two age groups. In young and healthy individuals, the injury results from highenergy trauma, whereas in the elderly population most of the fractures are osteoporotic resulting from a fall. With the increase in the aging population, there is also considerable growth in the number of pathological fractures and fractures around hip prosthesis (per prosthetic fractures).

'Financial or Other Competing Interest': None.

Submission 01-08-2017, Peer Review 17-09-2017,

Acceptance 23-09-2017, Published 09-10-2017.

Corresponding Author:

Dr. Santosh Mared,

Senior Resident,

Department of Orthopaedics,

Belagavi Institute of Medical Sciences,

Belagavi.

E-mail: santosh.mared@yahoo.com

DOI: $10.14260 /$ jemds $/ 2017 / 1237$ thick cortical bone with less vascularity and results in increased potential for healing disturbances.[2,3] Hence, subtrochanteric fracture is difficult to manage and associated with many complications.

Closed management of these proximal femoral fractures thus poses difficulties in obtaining and maintaining a reduction, making operative management the preferred treatment. The goal of operative treatment is restoration of normal length and angulation to restore adequate tension to the abductors.[3]

\section{The Obvious Advantages of Operative Treatment are-}

1. It avoids complications of prolonged bed rest and hospitalisation.

2. Accurate reduction and anatomical alignment.

3. Early mobilisation and weight bearing is possible with new implants and fixation technology.

The two primary options for treatment of proximal femoral fractures are intramedullary fixation and extramedullary fixation. Many internal fixation devices have been recommended, but because of high incidence of complications like non-union and implant failure, a series of evolution in designing a perfect implant has begun. ${ }^{[4,5]}$ 
Only recently better understanding of biology, reduction techniques and biomechanically improved implants like Gamma nail, Russell-Taylor nail and Proximal femoral nail allowed for these fractures to be addressed with consistent success. ${ }^{[6,7,8]}$ Fractures of the subtrochanteric region of femur can be difficult to treat. This anatomic region experiences the highest tensile and compressive stresses in the human skeleton. The proximal fragment is often quite short, offering limited opportunity for internal fixation which must withstand these substantial forces. In subtrochanteric fracture the proximal fragment is flexed, abducted and externally rotated due to the iliopsoas, abductor muscles and short external rotators muscle pull.

Hence, it is useful to perform an open reduction of the fracture fragments and then stabilise the proximal fragment to restore anatomical position prior to nail insertion.

\section{Objectives}

1. To study proximal femoral fractures.

2. To determine the rate of union, complications, operative risks and functional outcomes in proximal femoral fractures.

\section{MATERIALS AND METHODS}

The observational study consists of 20 adult patients (13 males and 7 females) with proximal femoral fractures of femur who were treated with PFN at BIMS, Belgaum, between Jan 2014 and March 2017. Patients were selected depending on admissions with subtrochanteric fracture in this period. The fractures were classified according to Russell and Taylor classification. ${ }^{9}$ Twenty cases were followed at regular intervals. This observational study was conducted with due emphasis for clinical observation and analysis of results after surgical management of proximal femoral fractures of femur with proximal femoral nail. Statistical analysis was performed using the SPSS statistical package, version 15.0 for Windows.

\section{Inclusion Criteria}

1. Age Group- above 20 yrs.

2. Male and female patients.

3. If fracture is $2.5 \mathrm{~cm}$ distal to lesser trochanter or even more proximal fractures if the lesser trochanter is intact.

4. Proximal $1 / 3^{\text {rd }}$ shaft femur fractures. Subtrochanteric fractures, in which lesser trochanter is not comminuted off proximal femur.

5. Consent to participate in the study.

\section{Exclusion Criteria}

1. Pathological fractures.

2. Multiple fractures.

3. Fractures in children.

4. Old neglected fractures.

\section{RESULTS}

Statistical Analysis of Operative Complications

\begin{tabular}{|c|c|c|}
\hline Intraoperative Complications & No. of Cases & Percentage \\
\hline Fracture of lateral cortex & 5 & 10 \\
\hline Failure to put derotation screw & 5 & 10 \\
\hline Jamming of nail & 1 & 2 \\
\hline Drill bit breakage & 2 & 4 \\
\hline \multicolumn{3}{|c|}{ Table 1. Intraoperative Complication } \\
\hline
\end{tabular}

\begin{tabular}{|c|c|c|}
\hline $\begin{array}{c}\text { Delayed } \\
\text { Complications }\end{array}$ & No. of Cases & Percentage \\
\hline Hip joint stiffness & 7 & 15 \\
\hline Knee joint stiffness & 2 & 5 \\
\hline Delayed union & 5 & 10 \\
\hline Shortening & 2 & 5 \\
\hline \multicolumn{2}{|c|}{ Table 2. Delayed Complications } \\
\hline
\end{tabular}

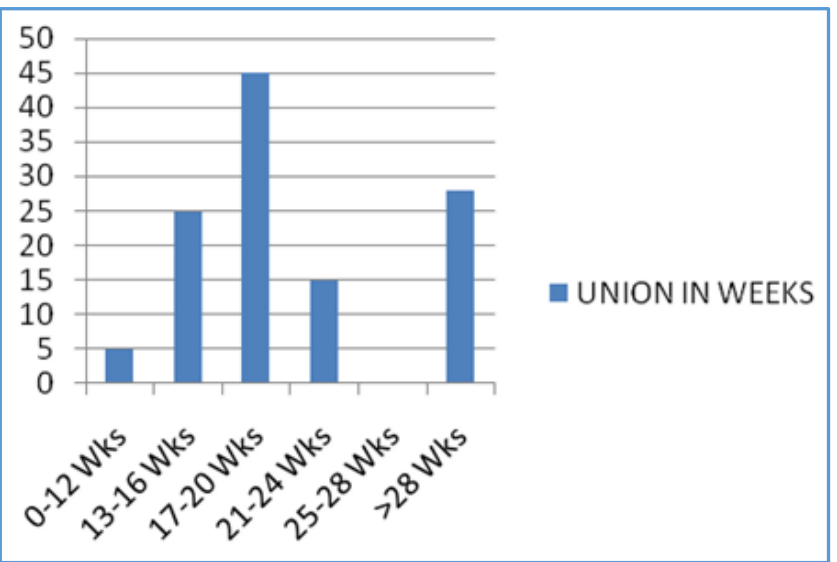

Graph showing Union of Fracture in Weeks

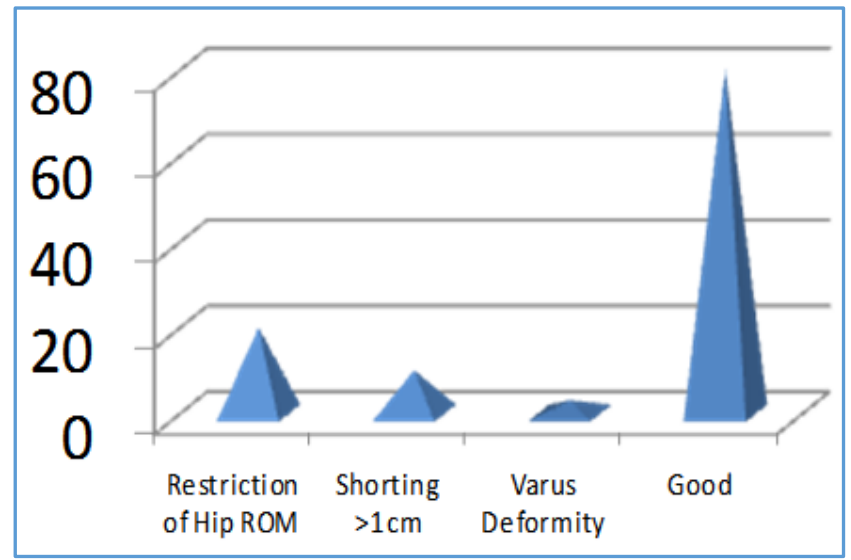

Graph showing Functional Outcome

\section{DISCUSSION}

Unlike osteoporotic trochanteric fractures, subtrochanteric fractures are usually the result of high-energy trauma and often subjected to a significant displacement and great difficulty in close reduction through traction. The high incidence of delayed union, malunion and non-union of fractures has left conservative treatment as advocated by DeLee et al, abolished in modem trauma care.[10,11,12] Extramedullary fixation with plating has the potential disadvantages of extensive surgical exposure, severe soft tissue damage and blood loss, thus leading to problems of fracture union and implant failure. In addition, the eccentrically plating is prone to fatigue breakage due to their mechanical load sharing effect.

Allowing a minimally open approach, intramedullary nailing is closely linked to "biological internal fixation," in addition to its mechanical benefits over plate fixation. Intramedullary fixation allows the surgeon to minimise soft tissue dissection, thereby reducing surgical trauma, blood loss, infection and wound complications.[13,5,9] A laboratory study (Mahomed et al, 1994) comparing a locked Gamma nail to a standard sliding hip screw for the fixation of stable and 
unstable subtrochanteric fractures showed that the intramedullary nail was more rigid and permitted less fracture displacement and concluded that intramedullary fixation was superior to extramedullary fixation. The currently used Gamma nail as an intramedullary device also has high learning curves with technical and mechanical failure rates of about $10 \%$ (collapse of the fracture area, cutout of the implant, fracture of the femur shaft) (Friedl 1996, Valverde et al 1998). The Gamma nail is susceptible to fail at its weakest point, the lag screw implant interface.[14,15,16,17,18]

The AO ASIF in 1996, therefore developed the Proximal Femoral Nail to reduce the risk of implant related complications. Therefore, in addition to the 8-mm load bearing femoral neck screw, the PFN has a $6.5 \mathrm{~mm}$ antirotation screw to increase the rotational stability of the neck fragment. An anatomic $6^{\circ}$ neck valgus bend in the coronal plane, a narrower distal diameter and distal flexibility of the nail eliminates the need for routine reaming of the femoral shaft and also minimises stress concentration and tension in the femoral shaft. This should reduce the risk of intraoperative and postoperative femoral shaft fractures.

PFN also has all the advantages of an intramedullary device such as decreasing the moment arm, can be inserted by closed technique which retains the fracture hematoma, decreases blood loss, minimises soft tissue dissection and wound infections. In an experimental study, Gotze et al (1998) compared the load ability of osteosynthesis of unstable per- and sub-trochanteric fractures and found that the PFN could bear the highest loads of all devices. Since its introduction in 1997, several clinical studies have shown good result (with few intraoperative problems and low rates of complications).[10,11,12]

The aim of our study was to assess the epidemiology and functional outcomes of subtrochanteric fractures with this newer method of intramedullary fixation with proximal femoral nail. We assessed the results with respect to intraoperative details, postoperative results and functional outcome.

By virtue of its load-sharing characteristics, the shorter lever on the proximal fixation and its biologically friendly implantation techniques, intramedullary nail fixation of subtrochanteric fractures has resulted in high union rates.

Although, biomechanically and biologically superior to plate fixation, closed nailing techniques can be technically difficult as a result of the position of the proximal fragment which is flexed, abducted and externally rotated. Awkward starting trajectories have often resulted in eccentric nail channels in the proximal fragment. Strafing points were often too lateral, resulting in varus alignment of the proximal fragment.

Hence, by opening the fracture site and bringing the proximal fragment in anatomical position w.r.t. distal fragment and subsequent nailing results in high bone union rates without any rotational or angular deformities.

Lei-Sheng Jiang et al in his study has no complications such as cut-out or breakage of the implants or pen-implant fractures. ${ }^{1}$ He recommended that the lag screw of PFN should be placed in the lower part of the femoral neck close to the femoral calcar with screw tip reaching the subchondral bone 5 to $10 \mathrm{~mm}$ below the articular cartilage in antero-posterior view. In lateral view, it should be placed in the centre of the femoral neck. There the lag screw will be definitely placed in the area of best bone quality. In 2002, Inger B Schipper in his study on biomechanical evaluation of PFN also concluded that if the hole through the nail of the hip pin was modified to a slot, there is significant reduction of axial loads on hip pin thereby reducing the cut-out risk. ${ }^{6}$

Werner et al was the first who introduced the term Zeffect, detected in 5 (7.1\%) of 70 cases. The incidence of cutout of the neck screw in this study was $8.6 \%$. The Z-effect phenomenon is referred as a characteristic sliding of the proximal screws to opposite directions during the postoperative weight-bearing period. ${ }^{18}$

The reverse Z-effect described by Boldin et al occurred with movement of the hip pin towards the lateral side, which required early removal. The mechanism is similar, but here the hip pin is sliding back, whereas the neck screw remains impacted to the hole of the nail. In their prospective study of 55 patients with unstable intertrochanteric or subtrochanteric fractures, they had 3 cases with Z-effect and 2 with reverse Z-effect. The authors in an effort to prevent the Z-effect phenomenon suggest the use of a "ring" in the lateral side of the hip pin.

The most recent study evaluating the use of PFN is from Fogagnolo et al, who reported 46 patients with an average rate of intraoperative technical or mechanical complications of $23.4 \%$. They also reported 2 implant failures and 1 fracture below the tip of the nail.

Daniel FA Menzes et al and Axel Gamulin (2005) in a clinical study of 155 consecutive patients treated with proximal femoral nail reported failure of fixation in $2 \%$, femoral shaft in $0.7 \%$, fixation failures included one cut out, one delayed fracture healing and one lateral displacement of the anti-rotation screw. Christian Boldin et al in his study of 55 patients of proximal femoral fractures with PFN noted 3 cases with Z-effect and 2 patients with reverse Z-effect; 2 patients had screw cut-out without any relation to fracture pattern and $10 \%$ of open reduction. $[19,20]$

Simmermacher et al (1999), in a clinical multicentre study reported technical failures of the PFN after poor reduction, malrotation or wrong choice of screws in $5 \%$ of the cases. A cut-out of the neck screw occurred in $0.6 \%$.[21,22,23,24]

In our study of subtrochanteric fractures treated with PFN, we encountered intraoperative complications like iatrogenic fracture of lateral cortex in 2 cases $(10 \%)$ due to selection of wrong entry point, failure to put derotation screw in 2 cases (10\%) and guidewire breakage in 1 case (5\%). Delayed complications like hip joint stiffness in 3 cases (15\%), knee joint stiffness in 1 case (5\%) and shortening of more than $1 \mathrm{~cm}$ in 1 case (5\%) and delayed union in 2 cases (10\%).

In our study, mean frequency of radiation exposure were 140 secs and mean duration of operation is 105 minutes and mean blood loss was $300 \mathrm{~mL}$.

Iatrogenic fracture of lateral cortex was seen in 2 cases, 2 cases of anti-rotation screw could not be put. Postoperatively, 2 of our cases had superficial infection. Mean duration of hospital stay was 24 days in and mean time of full weight bearing was 14 weeks in our study.

In our 20 cases, excellent results were seen in $45 \%$, good in $35 \%$ cases, fair in $15 \%$ cases and poor in $5 \%$ cases by PFN by open method. 


\section{CONCLUSION}

Subtrochanteric femoral fractures are usually treated surgically. In the last decade, extramedullary methods of fixation with various angular plates or with a compression hip screw with a plate are more and more replaced by newer intramedullary techniques because of their advantages: The surgical procedure is faster, the blood loss is smaller, the bone healing mainly remains in the reduced position with a biomechanically strong fixation that allows earlier weight bearing on the bone with less local and general

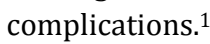

The abundant muscles around the subtrochanteric region usually cause significant displacement of the fractured fragments, leading to great difficulties in close reduction under traction. Open clamp assisted reduction through an incision at the fracture site restores the normal anatomical alignment and prevents any rotational/ angular deformities. The use of appropriate nail length and proper positioning of the nail and screws are necessary to avoid failure or revision. ${ }^{25}$ Most of the complications of proximal femoral nailing are surgeon and instruments related, which can be cut down by proper patient selection and good preoperative planning and restoring the anatomical alignment. Because of increasing occurrence of subtrochanteric fractures in younger age active males, higher demand is placed on treating surgeon to restore near normal function of leg.4

Osteosynthesis with the proximal femoral nail offers the advantages of high rotational stability of the head-neck fragment. Proximal femoral nail has the advantage of collapse at fracture site and is biomechanically sound as it is an intramedullary device. ${ }^{1}$ Postoperatively, early mobilisation can be begun as the fixation is rigid.

\section{PFN being an Intramedullary Load Sharing Device offers} better Biomechanical Stability-

1. Early weight bearing, better range of movements and early postoperative mobilisation are distinct advantages of PFN in subtrochanteric fractures.

2. Union rates through open technique are high, since better reduction is achieved and option of bone grafting is present in comminuted fractures.

3. In our study, there were no cases with screw cut-out, Zeffect, reverse Z-effect and implant failure or femoral fracture below the tip in any case.

4. Average duration of surgery was $105 \mathrm{~mm}$, which is more when compared with other standard studies since we used open technique. However, rate of infection and other operative complications were same as cases treated with closed reduction. But reduction achieved was better.

5. However, no cases of significant limb length discrepancy or varus deformities were seen in our study.

\section{Limitation of the Study is Low Sample Size}

Hence, we conclude that open reduction offer advantage in preventing malalignment of subtrochanteric fractures as compared to closed reduction. From our study, we conclude that PFN is a reliable implant for subtrochanteric fractures leading to high rate of bone union restoring the anatomical alignment and reduced chance of implant failure or deformities. Intramedullary fixation has biological and biomechanical advantages, but the operation is technically demanding. Gradual learning and great patience is needed in order to make this method truly successful.

\section{REFERENCES}

[1] Bucholz RW, F1ëckrnan JD, Court-Brown CM. Rockwood and Green's "Fractures in Adults". $6^{\text {th }}$ edn. 2009;2:1827-44.

[2] Lavelle DG. Fractures and dislocations of the hip. In: Canale ST, (eds). Campbell's operative orthopaedics. Chapter 52. Vol 3. 11 th edn. 2008:3237-308.

[3] McKibbin B. The biology of fracture healing in long bones. J Bone Joint Surg Br 1978;60-B(2):150-62.

[4] Mclaunn TM, Lawler EA. Treatment modalities for subtrochanteric fractures in elderly. Techniques in orthopaedics. Vol 19. Philadelphia: Lippincott Williams and Wilkins Inc. 2004:197-213.

[5] Hinton RY, Smith GS. The association of age, race and sex with the location of proximal femoral fractures in the elderly. J Bone Joint Surg Am 1993;75(5):752-9.

[6] Van Meeteren MC, van Rief YE, Roukema JA, et al. Condylar plate fixation of subtrochanteric femoral fractures. Injury 1996;27(10):715-7.

[7] Berman AT, Metzger PC, Bosacco SJ, et al. Treatment of the subtrochanteric fracture with the compression hip nail-a review of 138 consecutive cases. Orthop Trans 1979;3:225-56.

[8] Ruff ME, Lubbers LM. Treatment of subtrochanteric fractures with a sliding screw-plate device. J Trauma 1986;26(1):75-80.

[9] Boyd HB, Griffin LL. Classification and treatment of trochanteric fractures. Arch Surg 1949;58(6):853-66.

[10] Fielding JW, Magliato HJ. Subtrochanteric fractures. Surg Gynecol Obstet 1966;122(3):555-60.

[11] Zickel RE. Subtrochanteric femoral fractures. Orthop Clin North Am 1980;11(3):555-68.

[12] Habernek H, Wallner T, Aschauer E, et al. Comparision of ender nails, dynamic hip screw and gamma nails in treatment of peritrochanteric femoral fractures. Orthopedics 2000;23(2):121-7.

[13] DeLee JC, Clanton TO, Rockwood CA. Closed treatment of subtrochanteric fractures of the femur in a modified cast-brace. J Bone Joint Surg Am 1981;63(5):773-9.

[14] Velasco RU, Comfort TH. Analysis of treatment problems in subtraochanteric fractures of the femur. J Trauma 1978;18(7):513-23.

[15] Koval K, Zuckerman J. Hip fractures-a practical guide to management. Springer Science and Business Media 2000:191-252.

[16] Waddell JP. Subtrochanteric fractures of the femur: a review of 130 patients. J Trauma 1979;19(8):582-92.

[17] Seinsheimer F. Subtrochanteric fracture of the femur. J Bone Joint Surg Am 1978;60(3):300-6.

[18] Whatley JR, Garland DE, Whitecloud T, et al. Subtrochanteric fractures of the femur: treatment with ASIF blade plate fixation. South Med J 1978;71(11):1372-5.

[19] Asher MA, Tippett JW, Rockwood CA, et al. Compression fixation of subtrochanteric fractures. Clin Orthop Relat Res 1976;117:202-8.

[20] Jones W. Injuries of the thigh. Chapter 30 in WatsonJones fractures and joint injuries. $6^{\text {th }}$ edn. vol 2, 1982:999-1003. 
[21] Koch JC. The laws of bone architecture. Developmental Dynamics 1917;21(2):177-298.

[22] Chapman MW. The role of intramedullary nailing in fracture management. In: Browner BD, Edwards CD, (eds). The science and practice of intramedullary nailing. Philadelphia: Lea \& Febiger, 1987:17-23.

[23] Chapman MW, Bowman WE, Csongradi JJ, et al. The use of enders pins in extracapsular fractures of the hip. J Bone Joint Surg Am 1981;63(1):14-28.
[24] Jensen JS, Sonne-Holm S. Critical analysis of ender nailing in the treatment of trochanteric fractures. Acta Orthop Scand 1980;51(5):817-25.

[25] Taylor C, Russell T, Lavelle D. Intramedullary nailing of complex subtrochanteric fractures of the femoral shaft. Orthop Trans 1991;5(2):230. 